\title{
High-density quantum sensing with dissipative first order transitions
}

\author{
Meghana Raghunandan, ${ }^{1}$ Jörg Wrachtrup, ${ }^{2}$ and Hendrik Weimer ${ }^{1, *}$ \\ ${ }^{1}$ Institut für Theoretische Physik, Leibniz Universität Hannover, Appelstraße 2, 30167 Hannover, Germany \\ 23. Physikalisches Institut, Universität Stuttgart, Pfaffenwaldring 57, 70569 Stuttgart
}

\begin{abstract}
The sensing of external fields using quantum systems is a prime example of an emergent quantum technology. Generically, the sensitivity of a quantum sensor consisting of $N$ independent particles is proportional to $\sqrt{N}$. However, interactions invariably occuring at high densities lead to a breakdown of the assumption of independence between the particles, posing a severe challenge for quantum sensors operating at the nanoscale. Here, we show that interactions in quantum sensors can be transformed from a nuisance into an advantage when strong interactions trigger a dissipative phase transition in an open quantum system. We demonstrate this behavior by analyzing dissipative quantum sensors based upon nitrogen-vacancy defect centers in diamond. Using both a variational method and numerical simulation of the master equation describing the open quantum many-body system, we establish the existence of a dissipative first order transition that can be used for quantum sensing. We investigate the properties of this phase transition for two- and three-dimensional setups, demonstrating that the transition can be observed using current experimental technology. Finally, we show that quantum sensors based on dissipative phase transitions are particularly robust against imperfections such as disorder or decoherence, with the sensitivity of the sensor not being limited by the $T_{2}$ coherence time of the device. Our results can readily be applied to other applications in quantum sensing and quantum metrology where interactions are currently a limiting factor.
\end{abstract}

The challenges associated with quantum sensing within interacting systems is particularly relevant for magnetic field sensing with nitrogen-vacancy (NV) color centers in diamond, as strong magnetic dipole interactions present a challenge to perform magnetometry at high densities [1]. For NV centers, performing magnetometry at high densities is particularly important, enabling to study processes inside living cells [2]. These challenges imposed by interacting systems are not totally surprising, given that the magnetic dipole moments of NV centers is what enables to measure magnetic fields in the first place. Hence, the effect we are addressing is quite generic and is also found in related applications; for example, uncertainties caused by interactions are currently one of the most important limiting factors for optical lattice clocks $[3,4]$.

Building on the tremendous progress in controlling individual [5-12] and interacting [13-15] NV centers, combined with the first studies investigating many-body effects [16-22], we consider large ensembles of microwavedriven NV centers interacting via the magnetic dipole interaction. As an important ingredient, we also incorporate optical pumping of the NV centers towards the $m_{s}=0$ spin state, see Fig. 1. Such driven-dissipative spin systems are closely related to dissipative Ising models studied in Rydberg gases [23, 24], which exhibit a dissipative first order liquid-gas transition at a critical strength of the driving field [25-27], with the first order transition line ending in a critical point belonging to the Ising universality class [24]. Crucially, the susceptibility of the system diverges with the number of spins at the transition point, showing a dramatic response of the system that can be used for quantum sensing [28]. A key advantage of turning to the steady state of a drivendissipative system is that all additional imperfections, such as disorder or decoherence, can be integrated into the sensing process, meaning they only shift the position of the transition without affecting its usefulness for quantum sensing applications.

In this Letter, we demonstrate that the dissipative phase transition is also present in the case of NV centers. Focusing first on the case of two-dimensional arrays of $\mathrm{NV}$ centers, we perform a variational analysis of the many-body system in thermodynamic limit. We compare the variational results to wave-function MonteCarlo simulations for systems containing up to 20 spins, which to our knowledge, is the largest number of spins treated so far in an open quantum many-body systems while retaining the full Hilbert space. We show that in three-dimensional systems, the anisotropy of the dipoledipole interactions replaces the sharp phase transition by a smooth crossover, however, the transition can easily be restored by applying a magnetic field gradient of modest strength. Finally, we address the role of additional imperfections and decoherence channels within the setup and demonstrate that a finite $T_{2}$ coherence time does not limit the sensitivity of the quantum sensor.

In our investigations, we consider a system of $N$ NV centers in a lattice geometry. Such structures can be implemented using targeted ion implantation at the nanometer scale [29]. Furthermore, the NV centers can be preferentially aligned along the axis of the external magnetic field [30]. We consider an effective two-level description of the NV centers, where the $m_{s}=-1$ state is off-resonant with respect to the microwave field, see Fig. 1, due to the external bias field $B_{0}$. In the rotating frame of the driving, the Hamiltonian is of the form

$$
H=\frac{\hbar \Delta}{2} \sum_{i}^{N} \sigma_{z}^{(i)}+\frac{\hbar \Omega}{2} \sum_{i}^{N} \sigma_{x}^{(i)}+\sum_{i<j}^{N} V_{i j}
$$


(a)

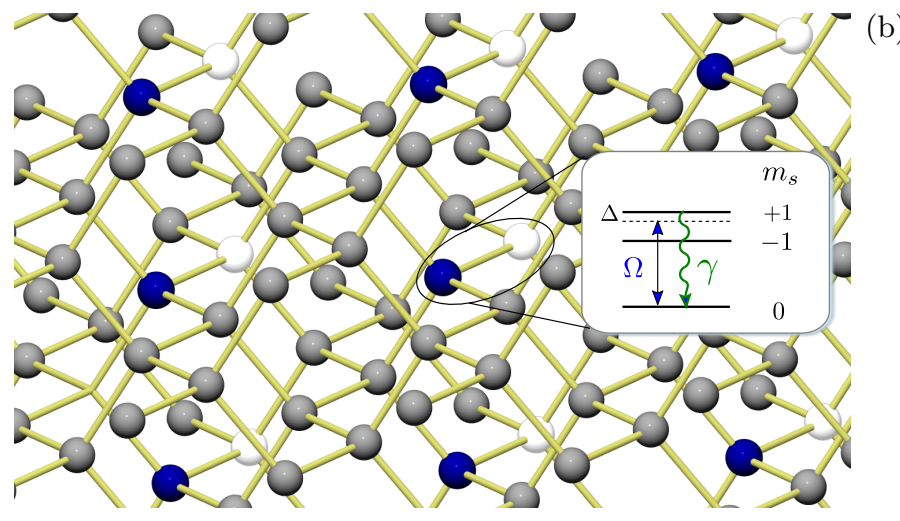

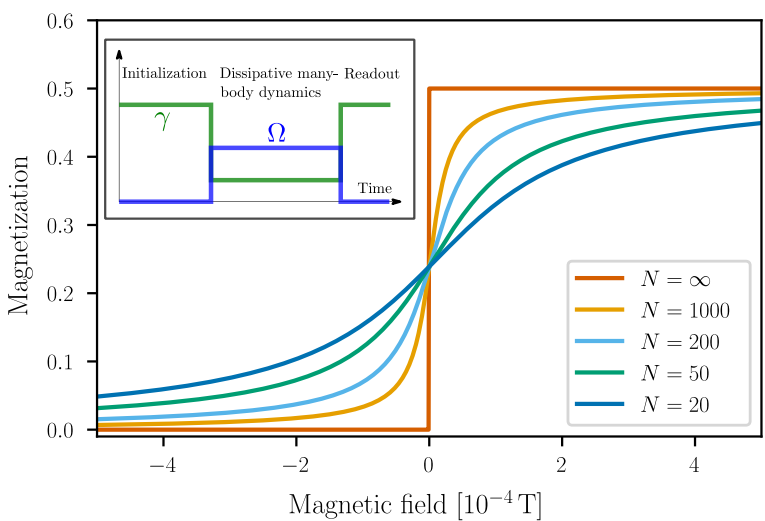

FIG. 1. Setup of the system for dissipative quantum sensing. (a) Many-body system of nitrogen-vacancy centers in diamond showing individual carbon atoms (gray) and nitrogen impurities (blue) accompanied by a vacancy site (white). The electronic ground state is a triplet state that is split by an external bias field. (b) Sketch of the dimensionless magnetization of the system across a first order phase transition. The response of the system strongly increases for larger system sizes. At the transition, the derivative of the magnetization is proportional to $\sqrt{N}$. The inset shows the sensing protocol consisting of NV initialization, dissipative many-body dynamics, and readout of the NV spin state.

where $\Delta$ is the detuning from the electron spin resonance and $\Omega$ is the Rabi frequency of the microwave driving. The dipole-dipole interaction $V_{i j}$ is given by

$$
\begin{aligned}
V_{i j} & =\left(1-3 \cos ^{2} \vartheta_{i j}\right) \frac{\mu^{2}}{\left|\mathbf{r}_{i}-\mathbf{r}_{j}\right|^{3}} \\
& \times\left\{\frac{1}{4}\left[1+\sigma_{z}^{(i)}\right]\left[1+\sigma_{z}^{(j)}\right]-\sigma_{+}^{(i)} \sigma_{-}^{(j)}-\sigma_{-}^{(i)} \sigma_{+}^{(j)}\right\},
\end{aligned}
$$

where $\mathbf{r}_{i}$ denotes the position of the NVs, $\mu$ indicates the magnetic dipole moment, and $\vartheta_{i j}$ is the angle between the NV axis and the vector connecting sites $\mathbf{r}_{i}$ and $\mathbf{r}_{j}$. We account for the optical pumping of the spins by considering a quantum master equation in Lindblad form,

$$
\frac{d}{d t} \rho=-\frac{i}{\hbar}[H, \rho]+\sum_{i}^{N} \gamma\left(\sigma_{-}^{(i)} \rho \sigma_{+}^{(i)}-\frac{1}{2}\left\{\sigma_{+}^{(i)} \sigma_{-}^{(i)}, \rho\right\}\right)
$$

where $\gamma$ is the rate of the optical pumping process, which can be controlled by the strength of the green pump laser. In all our calculations, we assume the NV centers to be separated by $r=5 \mathrm{~nm}$, and the optical pumping rate to be $\gamma=1 \mathrm{MHz}$. Unless we specifically investigate the response to an additional magnetic field, we assume the driving to be on resonance, i.e., $\Delta=0$.

As in the case of conventional NV sensors [1], the system is read out by the fluorescence signal from the NV centers in the $m_{s}=1$ state. The only difference is that the dynamics of the system does not follow a Ramsey sequence, but is governed by the dissipative many-body dynamics of the quantum master equation.

Two-dimensional systems. - We first turn to the analysis of two-dimensional square lattices where the dipoles are oriented perpendicular to the plane of the system. We also simplify the analysis by considering only interactions between adjacent lattice sites; taking the full long-range tail into account only slightly modifies our results on a quantitative level, but the qualitative findings will remain unchanged [31] As a first step, we investigate the steady state of the quantum master equation based on the variational principle for open quantum systems [25]. Here, we use product states of the form $\rho=\prod_{i} \rho_{i}$ as our variational basis, with $\rho_{i}$ being the reduced density matrix at site $i$. Then, we find a first order transition of the NV magnetization $m=\sum_{i}\left(1+\left\langle\sigma_{z}^{(i)}\right\rangle\right) /(2 N)$ in the driving strength $\Omega$, see Fig. 2 . This transition appears to be closely related to what has been predicted for dissi-

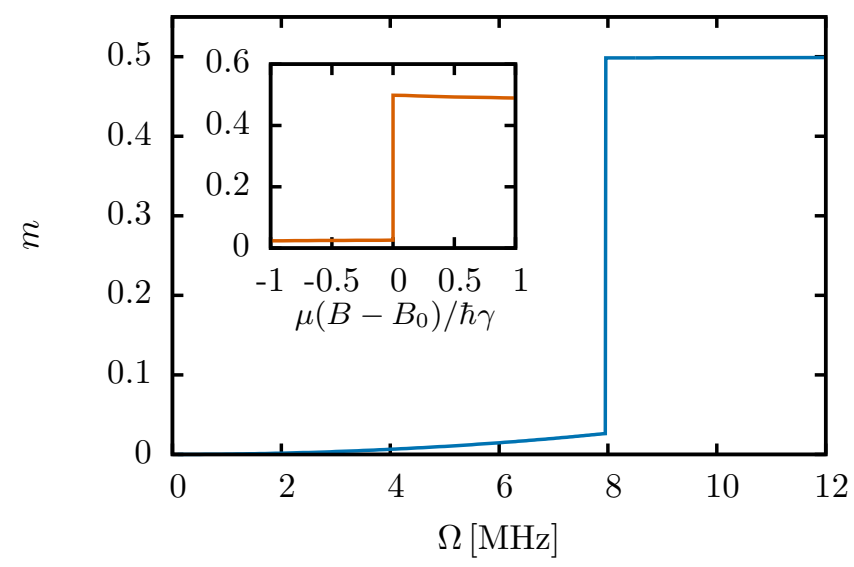

FIG. 2. Variational solution for the steady state magnetization showing a first order phase transition. The phase transition can be triggered by varying either the Rabi frequency $\Omega$ or the external magnetic field (inset) $(V=2 \pi \times 400 \mathrm{kHz}$, $\gamma=1 \mathrm{MHz}$ ). 


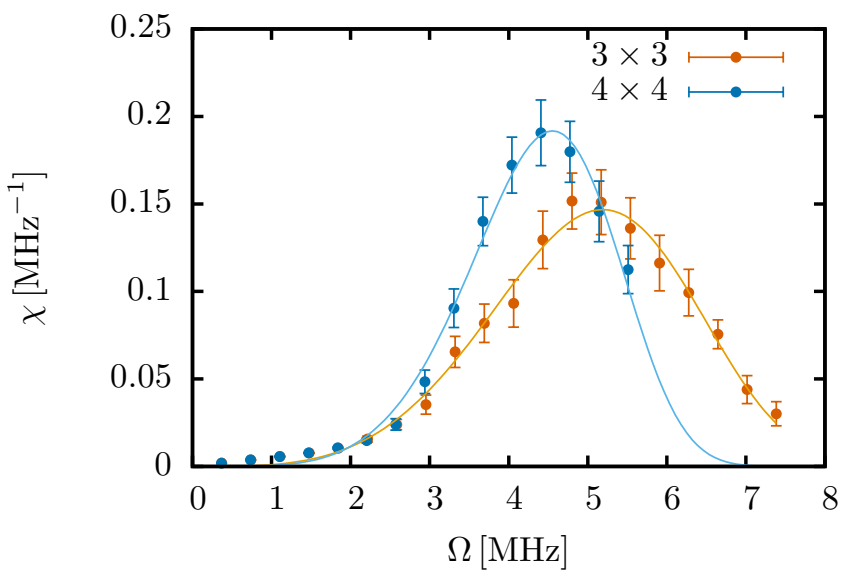

FIG. 3. Averaged results from 1000 wave-function MonteCarlo trajectories showing the steady state susceptibility $\chi$ for $3 \times 3$ and $4 \times 4$ geometries. The solid lines are fits to a Weibull distribution.

pative Ising models discussed in the context of Rydberg gases, where the flip-flop term of Eq. (3) is absent [32]. Crucially, the first order transition can also be triggered by modifying the external magnetic field, allowing to use this transition for the sensing of static fields.

Wave-function Monte-Carlo simulations. - We perform numerical simulations of the full quantum master equation for systems up to 20 spins. We use the results from the simulations based on a wave-function MonteCarlo approach [33], which we extended to a massively parallelized version, to serve as a benchmark for our variational analysis. In particular, we are interested in the existence of the first order transition predicted by the variational approach. For this, we investigate the magnetic susceptibility $\chi=\partial m / \partial \Omega$, which diverges at a first order transition. Figure 3 shows the numerically obtained susceptibility for different system sizes. Interestingly, we find that the susceptibility data closely follows a Weibull distribution $\chi(\Omega) \sim \Omega^{k-1} \exp \left[-(\Omega / \lambda)^{k}\right]$. We note that the Weibull distribution has been discussed in the context of the relaxation from metastable states, with the parameter $k$ controlling their relative decay rates $[34,35]$. Such metastable states also play an important role in dissipative Ising models $[36,37]$. To investigate the scaling with the number of spins $N$ in detail, we turn to a finite size scaling analysis. For this, we aim to describe the simulation results for the susceptibility peak in terms of a scaling function, from which we can extract how the susceptiblity peak changes with $N$. Here, we also include anisotropic geometries to be able to treat larger system sizes up to 20 spins. Our ansatz for the scaling function is given by

$$
\chi=c N^{\alpha} \tilde{\chi}(\lambda),
$$

where $\lambda=N_{x} / N_{y}$ is the anisotropy given in terms of the number of spins in the $x$ and $y$ direction, respectively,

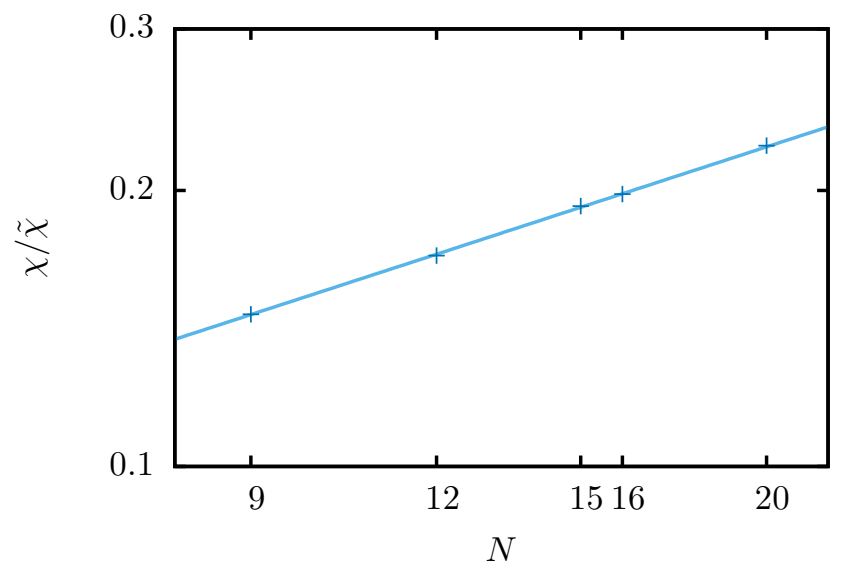

FIG. 4. Finite size scaling of the peak of the reduced susceptibility $\chi / \tilde{\chi}$. The collapse of the data onto a single line in the logarithmic plot shows that the susceptibility diverges with the system size $N$. The solid line is an algebraic fit to the data.

while $c$ and $\alpha$ are numerical constants [38]. Crucially, when the exponent $\alpha$ is found to be positive, the susceptibility diverges with $N$, signalling the presence of a first order transition. The reduced scaling function $\tilde{\chi}$ captures the effects of anisotropic system sizes and must satisfy the conditions $\tilde{\chi}(\lambda)=\tilde{\chi}(1 / \lambda)$ and $\tilde{\chi}(1)=1$. Consequently, we can perform a series expansion according to $\tilde{\chi}(\lambda)=1+d[\log \lambda]^{2}+O\left(\log [\lambda]^{4}\right)$, which we can truncate for not too large anisotropies. $d$ is another numerical constant that can be determined from fitting to the simulation data. Then, the reduced susceptibility $\chi / \tilde{\chi}=c N^{\alpha}$ should be given by a simple algebraic function. Fig. 4 demonstrates that this is indeed the case, showing that the ansatz of Eq. (4) is correct, confirming the existence of the first order transition. The observed exponent $\alpha=0.527 \pm 0.006$ shows that the system exhibits basically the same scaling of the sensitivity for quantum sensing as a noninteracting ensemble $(\alpha=1 / 2)$.

Three-dimensional systems. - As the next step, we will study the properties of the system in three spatial dimensions. This will be especially important as controlling the implantation depth of the NV centers will be particularly challenging, making it natural to focus on effectively three-dimensional setups. Here, we turn to a threedimensional cubic lattice to investigate the consequences. In particular, the anisotropy of the dipole-dipole interaction will now play an important role. Crucially, the dipole-dipole interaction vanishes when integrated over the full solid angle, as the ferromagnetic and antiferromagnetic contributions exactly cancel each other. To capture this property in our nearest-neighbor model, we set the interaction energy within the plane of the dipoles to $V=\mu^{2} / r^{3}$ and to $-2 V$ in the third direction.

In three dimensions, the system sizes are prohibitively large for wave-function Monte-Carlo simulations. There- 


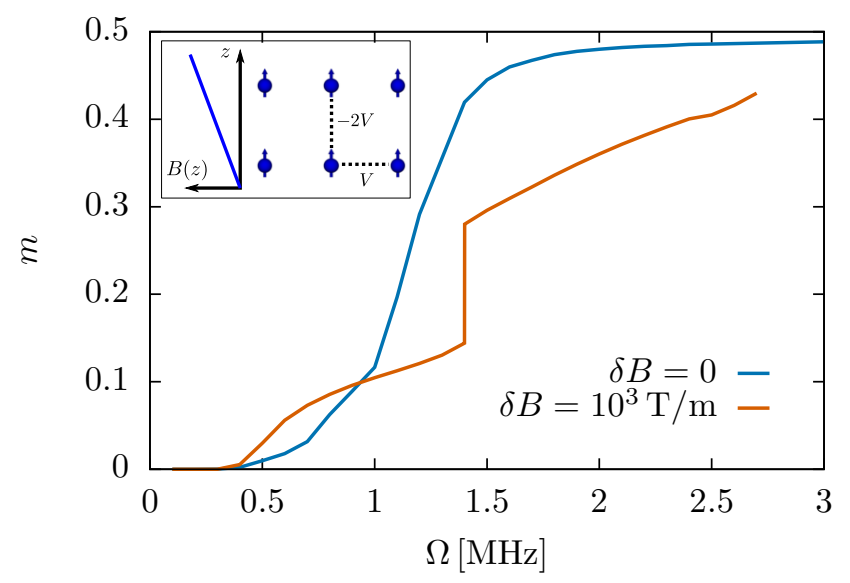

FIG. 5. Variational solution for the magnetization of the central layer in a three-dimensional system. The first order transition is replaced by a smooth crossover. Using a magnetic field gradient (inset), the phase transition can be restored.

fore, we restrict our analysis to the variational approach, noting that in larger dimensions, the variational solution is even closer to the exact steady state [39]. Here, we consider a system consisting of three two-dimensional layers, with the central layer being at the zero point of the magnetic field gradient. Within the variational analysis, we find that the other two layers are almost completely polarized in the $m_{s}=0$ state, i.e., adding additional layers will not change the results. Additionally, we find that the anisotropy of the dipole-dipole interaction replaces the first order transition by a smooth crossover, see Fig. 5 . Nevertheless, it is possible to recover the transition by applying a magnetic field gradient along the NV axis, effectively breaking the symmetry of the dipolar interaction. The first order transition appears already for quite modest field gradients on the order of $\delta B=10^{3} \mathrm{~T} / \mathrm{m}$, which are readily achievable in experiments. For larger values of the gradient, the first order jump in the magnetization will be even more pronounced, eventually recovering the $2 \mathrm{D}$ results for very strong gradients. This underlines the usefulness of dissipative quantum sensing even for three-dimensional systems.

Decoherence and other imperfections. - So far, our analysis has been based on a rather idealized setup. In any real diamond sample, there will be several sources of imperfections related to decoherence or to disorder from imperfect positioning of the NV centers. First, we want to point that disorder in the NV interaction energies or missing sites in the lattice due to off-axis NV centers are not going to play an important role. Crucially, these imperfections only affect the strengths of the coupling constants, but cannot reverse their signs. From the analysis of random-bond Ising models [40], it is known that the underlying phase transition is robust against such a type of disorder, which is consistent with our numerical simu-

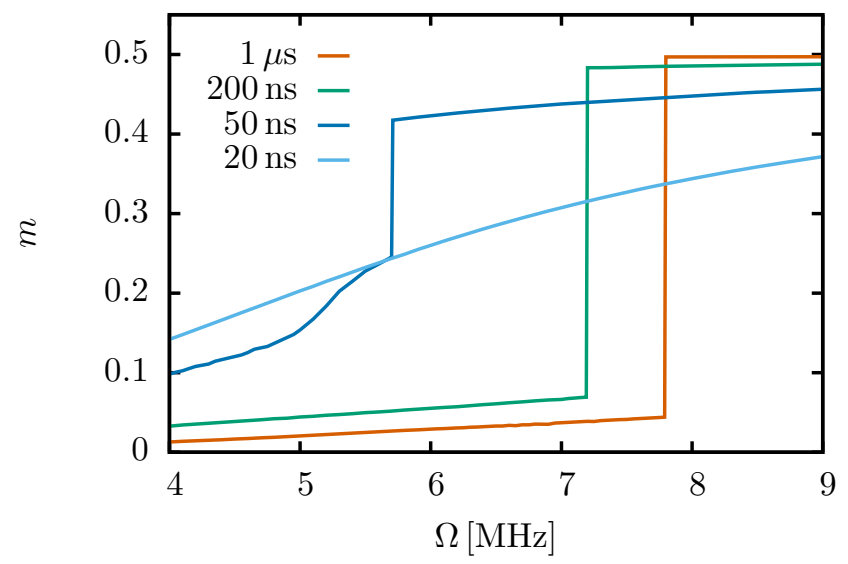

FIG. 6. Consequences of additional decoherence channels for different $T_{2}$ times. The first order transition is particularly robust to these additional decoherence processes, even when their associated rates become larger than the strength of the dipole-dipole interaction between the NV centers. Only for very short $T_{2}$ times, the phase transition is replaced by a smooth crossover.

lations for disorder in the system [31]. This leaves decoherence processes caused by residual nitrogen impurities and ${ }^{13} \mathrm{C}$ nuclear spins as the dominant challenge. Hence, we investigate in detail how a limited $T_{2}$ time caused by these decoherence processes will affect the performance of the dissipative quantum sensor.

Within the variational analysis, we add additional jump operators $c_{i}=\sqrt{1 / T_{2}} \sigma_{z}^{(i)}$ to the quantum master equation. Importantly, we find that the existence of the first order transition is robust against quite strong decoherence rates, see Fig. 6. Crucially, the phase transition does not merely survive in a regime where the decoherence is perturbatively small compared to the dipoledipole interaction, but even in a regime where the decoherence rates are several times larger than the coherent interaction strength, which amounts to $V=2 \pi \times 400 \mathrm{kHz}$ at a NV distance of $r=5 \mathrm{~nm}$ in our case. We attribute this strong robustness against decoherence to the steady state being an effective thermal (but non-classical) state [41]. Such a state is diagonal in an appropriate energy eigenbasis, making it less susceptible to decoherence processes. The additional decoherence also leads to a shift in the transition point, requiring to characterize the coherence properties of a device before employing it as a quantum sensor. For more dilute NV samples, the global timescale of the system gets reduced, leading to a stronger susceptibility to decoherence. E.g., for a NV distance of $r=11 \mathrm{~nm}$, the phase transition will be replaced by a smooth crossover for $T_{2}$ at about $500 \mathrm{~ns}$ instead of $50 \mathrm{~ns}$. We would also like to point out that both the first order transition and the robustness to decoherence remain present without an external bias field $B_{0}[31]$. 
Finally, we estimate the sensitivity of the dissipative quantum sensor, which we can extract from the finite size scaling behavior of the susceptibility, as the change in fluorescense from the NV centers is proportional to the magnetic susceptibility [1]. Within our wave-function Monte-Carlo simulations, we find that the susceptibility for DC fields $\chi_{D C}=\partial m / \partial B$ shows very similar behavior as the AC susceptibility [31], so the DC and AC sensitivity of the sensor are essentially the same. This is very different in NV magnetometry using noninteracting ensembles, as there the $T_{2}^{*}$ limited DC sensitivity is generally worse than the $T_{2}$ limited AC sensitivity since DC sensing does not allow for dynamical decoupling techniques [1]. As our proposed sensor is not limited by $T_{2}^{*}$, we expect our approach to be particularly useful for sensing DC fields. We can infer the sensitivity of the dissipative sensor to be $\eta \approx 3 \mathrm{nT} \mathrm{Hz}^{-1 / 2}$ for $N=10^{3}$ spins and $\eta \approx 300 \mathrm{fT} \mathrm{Hz}^{-1 / 2}$ for $N=10^{11}$ [31]. This sensitivity is approximately a factor of three improvement over what has been recently demonstrated using large ensembles of noninteracting NV centers [42], while at the same time offering a much smaller sensor size. Additionally, we would like to stress that our dissipative quantum sensor can tolerate large decoherence rates and operate at very small sensor sizes. These unique features makes it extremely promising to use dissipative NV sensors in NV-rich nanodiamonds [43], e.g., for the investigation of biological processes inside living cells.

In summary, we have established a quantum sensing protocol based on dissipative phase transitions. We have demonstrated the usefulness of our approach for quantum sensing with nitrogen-vacancy defect centers in diamond, finding a strong resilience of our protocol against decoherence processes. Finally, our protocol does not depend on the microscopic details of the sensing process, allowing for an immediate transfer to other applications in quantum sensing (see [44] for a concrete example) and quantum metrology.

We thank D. D. B. Rao for fruitful discussions. This work was funded by the Volkswagen Foundation and the DFG within SFB 1227 (DQ-mat).

* hweimer@itp.uni-hannover.de

[1] L. Rondin, J.-P. Tetienne, T. Hingant, J.-F. Roch, P. Maletinsky, and V. Jacques, Magnetometry with nitrogen-vacancy defects in diamond, Rep. Prog. Phys. 77, 056503 (2014).

[2] G. Kucsko, P. C. Maurer, N. Y. Yao, M. Kubo, H. J. Noh, P. K. Lo, H. Park, and M. D. Lukin, Nanometre-scale thermometry in a living cell, Nature 500, 54 (2013).

[3] T. L. Nicholson, S. L. Campbell, R. B. Hutson, G. E. Marti, B. J. Bloom, R. L. McNally, W. Zhang, M. D. Barrett, M. S. Safronova, G. F. Strouse, W. L. Tew, and J. Ye, Systematic evaluation of an atomic clock at $2 \times 10^{-18}$ total uncertainty, Nature Commun. 6, 6896
(2015).

[4] I. Ushijima, M. Takamoto, M. Das, T. Ohkubo, and H. Katori, Cryogenic optical lattice clocks, Nature Photon. 9, 185 (2015).

[5] F. Jelezko, T. Gaebel, I. Popa, A. Gruber, and J. Wrachtrup, Observation of Coherent Oscillations in a Single Electron Spin, Phys. Rev. Lett. 92, 076401 (2004).

[6] L. Childress, M. V. Gurudev Dutt, J. M. Taylor, A. S. Zibrov, F. Jelezko, J. Wrachtrup, P. R. Hemmer, and M. D. Lukin, Coherent Dynamics of Coupled Electron and Nuclear Spin Qubits in Diamond, Science 314, 281 (2006).

[7] M. V. G. Dutt, L. Childress, L. Jiang, E. Togan, J. Maze, F. Jelezko, A. S. Zibrov, P. R. Hemmer, and M. D. Lukin, Quantum Register Based on Individual Electronic and Nuclear Spin Qubits in Diamond, Science 316, 1312 (2007).

[8] J. M. Taylor, P. Cappellaro, L. Childress, L. Jiang, D. Budker, P. R. Hemmer, A. Yacoby, R. Walsworth, and M. D. Lukin, High-sensitivity diamond magnetometer with nanoscale resolution, Nature Phys 4, 810 (2008).

[9] P. Neumann, N. Mizuochi, F. Rempp, P. Hemmer, H. Watanabe, S. Yamasaki, V. Jacques, T. Gaebel, F. Jelezko, and J. Wrachtrup, Multipartite Entanglement Among Single Spins in Diamond, Science 320, 1326 (2008).

[10] P. C. Maurer, G. Kucsko, C. Latta, L. Jiang, N. Y. Yao, S. D. Bennett, F. Pastawski, D. Hunger, N. Chisholm, M. Markham, D. J. Twitchen, J. I. Cirac, and M. D. Lukin, Room-Temperature Quantum Bit Memory Exceeding One Second, Science 336, 1283 (2012).

[11] N. Bar-Gill, L. Pham, A. Jarmola, D. Budker, and R. Walsworth, Solid-state electronic spin coherence time approaching one second, Nature Commun. 4, 1743 (2013).

[12] Y. Romach, C. Müller, T. Unden, L. J. Rogers, T. Isoda, K. M. Itoh, M. Markham, A. Stacey, J. Meijer, S. Pezzagna, B. Naydenov, L. P. McGuinness, N. Bar-Gill, and F. Jelezko, Spectroscopy of Surface-Induced Noise Using Shallow Spins in Diamond, Phys. Rev. Lett. 114, 017601 (2015).

[13] X. Zhu, S. Saito, A. Kemp, K. Kakuyanagi, S.-I. Karimoto, H. Nakano, W. J. Munro, Y. Tokura, M. S. Everitt, K. Nemoto, M. Kasu, N. Mizuochi, and K. Semba, Coherent coupling of a superconducting flux qubit to an electron spin ensemble in diamond, Nature 478, 221 (2011).

[14] F. Dolde, I. Jakobi, B. Naydenov, N. Zhao, S. Pezzagna, C. Trautmann, J. Meijer, P. Neumann, F. Jelezko, and J. Wrachtrup, Room-temperature entanglement between single defect spins in diamond, Nature Phys. 9, 139 (2013).

[15] J. Choi, S. Choi, G. Kucsko, P. C. Maurer, B. J. Shields, H. Sumiya, S. Onoda, J. Isoya, E. Demler, F. Jelezko, N. Y. Yao, and M. D. Lukin, Depolarization dynamics in a strongly interacting solid-state spin ensemble, arXiv:1608.05471 (2016).

[16] N. Y. Yao, L. Jiang, A. V. Gorshkov, Z.-X. Gong, A. Zhai, L.-M. Duan, and M. D. Lukin, Robust Quantum State Transfer in Random Unpolarized Spin Chains, Phys. Rev. Lett. 106, 040505 (2011).

[17] H. Weimer, N. Y. Yao, C. R. Laumann, and M. D. Lukin, Long-Range Quantum Gates using Dipolar Crystals, Phys. Rev. Lett. 108, 100501 (2012). 
[18] E. M. Kessler, G. Giedke, A. Imamoglu, S. F. Yelin, M. D. Lukin, and J. I. Cirac, Dissipative phase transition in a central spin system, Phys. Rev. A 86, 012116 (2012).

[19] H. Weimer, N. Y. Yao, and M. D. Lukin, Collectively Enhanced Interactions in Solid-State Spin Qubits, Phys. Rev. Lett. 110, 067601 (2013).

[20] J. Cai, A. Retzker, F. Jelezko, and M. B. Plenio, A largescale quantum simulator on a diamond surface at room temperature, Nat Phys 9, 168 (2013).

[21] A. Albrecht, G. Koplovitz, A. Retzker, F. Jelezko, S. Yochelis, D. Porath, Y. Nevo, O. Shoseyov, Y. Paltiel, and M. B. Plenio, Self-assembling hybrid diamondbiological quantum devices, New J. Phys. 16, 093002 (2014).

[22] S. Choi, J. Choi, R. Landig, G. Kucsko, H. Zhou, J. Isoya, F. Jelezko, S. Onoda, H. Sumiya, V. Khemani, C. von Keyserlingk, N. Y. Yao, E. Demler, and M. D. Lukin, Observation of discrete time-crystalline order in a disordered dipolar many-body system, arXiv:1610.08057 (2016).

[23] T. E. Lee, H. Häffner, and M. C. Cross, Antiferromagnetic phase transition in a nonequilibrium lattice of Rydberg atoms, Phys. Rev. A 84, 031402 (2011).

[24] M. Marcuzzi, E. Levi, S. Diehl, J. P. Garrahan, and I. Lesanovsky, Universal Nonequilibrium Properties of Dissipative Rydberg Gases, Phys. Rev. Lett. 113, 210401 (2014).

[25] H. Weimer, Variational Principle for Steady States of Dissipative Quantum Many-Body Systems, Phys. Rev. Lett. 114, 040402 (2015).

[26] H. Weimer, Variational analysis of driven-dissipative Rydberg gases, Phys. Rev. A 91, 063401 (2015).

[27] A. Kshetrimayum, H. Weimer, and R. Orus, A simple tensor network algorithm for $2 \mathrm{~d}$ steady states, arXiv:1612.00656 (2016).

[28] S. Gammelmark and K. Mølmer, Phase transitions and Heisenberg limited metrology in an Ising chain interacting with a single-mode cavity field, New J. Phys. 13, 053035 (2011).

[29] D. Scarabelli, M. Trusheim, O. Gaathon, D. Englund, and S. J. Wind, Nanoscale Engineering of CloselySpaced Electronic Spins in Diamond, Nano Lett. 16, 4982 (2016).

[30] T. Karin, S. Dunham, and K.-M. Fu, Alignment of the diamond nitrogen vacancy center by strain engineering, Appl. Phys. Lett. 105, 053106 (2014).

[31] See the Supplemental Material for a numerical investiga- tion of the effects of disorder and the full dipolar interaction, as well as a detailed discussion of the sensitivity of the proposed quantum sensor.

[32] H. Bernien et al., Probing many-body dynamics on a 51-atom quantum simulator, Nature 551, 579 (2017).

[33] J. Johansson, P. Nation, and F. Nori, QuTiP 2: A Python framework for the dynamics of open quantum systems, Comp. Phys. Comm. 184, 1234 (2013).

[34] R. S. Maier and D. L. Stein, Limiting Exit Location Distributions in the Stochastic Exit Problem, SIAM J. Appl. Math. 57, 752 (1997).

[35] D. A. Adams, L. M. Sander, and R. M. Ziff, The barrier method: A technique for calculating very long transition times, J. Chem. Phys. 133, 124103 (2010).

[36] D. C. Rose, K. Macieszczak, I. Lesanovsky, and J. P. Garrahan, Metastability in an open quantum Ising model, Phys. Rev. E 94, 052132 (2016).

[37] F. Letscher, O. Thomas, T. Niederprüm, M. Fleischhauer, and H. Ott, Bistability vs. Metastability in Driven Dissipative Rydberg Gases, arXiv:1611.00627 (2016).

[38] K. Binder and J. S. Wang, Finite-size effects at critical points with anisotropic correlations: Phenomenological scaling theory and Monte Carlo simulations, J. Stat. Phys. 55, 87 (1989).

[39] H. Weimer, Tailored jump operators for purely dissipative quantum magnetism, J. Phys. B 50, 024001 (2017).

[40] K. Binder and A. P. Young, Spin glasses: Experimental facts, theoretical concepts, and open questions, Rev. Mod. Phys. 58, 801 (1986).

[41] M. F. Maghrebi and A. V. Gorshkov, Nonequilibrium many-body steady states via Keldysh formalism, Phys. Rev. B 93, 014307 (2016).

[42] T. Wolf, P. Neumann, K. Nakamura, H. Sumiya, T. Ohshima, J. Isoya, and J. Wrachtrup, Subpicotesla Diamond Magnetometry, Phys. Rev. X 5, 041001 (2015).

[43] L.-J. Su, C.-Y. Fang, Y.-T. Chang, K.-M. Chen, Y.-C. $\mathrm{Yu}$, J.-H. Hsu, and H.-C. Chang, Creation of high density ensembles of nitrogen-vacancy centers in nitrogenrich type Ib nanodiamonds, Nanotechnology 24, 315702 (2013).

[44] C. G. Wade, M. Marcuzzi, E. Levi, J. M. Kondo, I. Lesanovsky, C. S. Adams, and K. J. Weatherill, Terahertz-driven phase transition applied as a room-temperature terahertz detector, arXiv:1709.00262 (2017). 


\title{
Supplemental Material for "High-density quantum sensing with dissipative first order transitions"
}

\author{
Meghana Raghunandan, ${ }^{1}$ Jörg Wrachtrup, ${ }^{2}$ and Hendrik Weimer ${ }^{1}$ \\ ${ }^{1}$ Institut für Theoretische Physik, Leibniz Universität Hannover, Appelstraße 2, 30167 Hannover, Germany \\ 2. Physikalisches Institut, Universität Stuttgart, Pfaffenwaldring 57, 70569 Stuttgart
}

\section{EFFECT OF LONG-RANGE INTERACTIONS AND DISORDER}

We analyze the effects of long-range dipolar interactions and disorder caused by vacancies in our system. We compare the following three cases to the simplified case of nearest neighbour interaction for a $3 \times 3$ geometry: i. full long-range dipolar interactions, ii. disorder caused by a vacany in the lattice with only nearest neighbour interactions, and, iii. disorder caused by a vacany in lattice with full long-range dipole interactions.

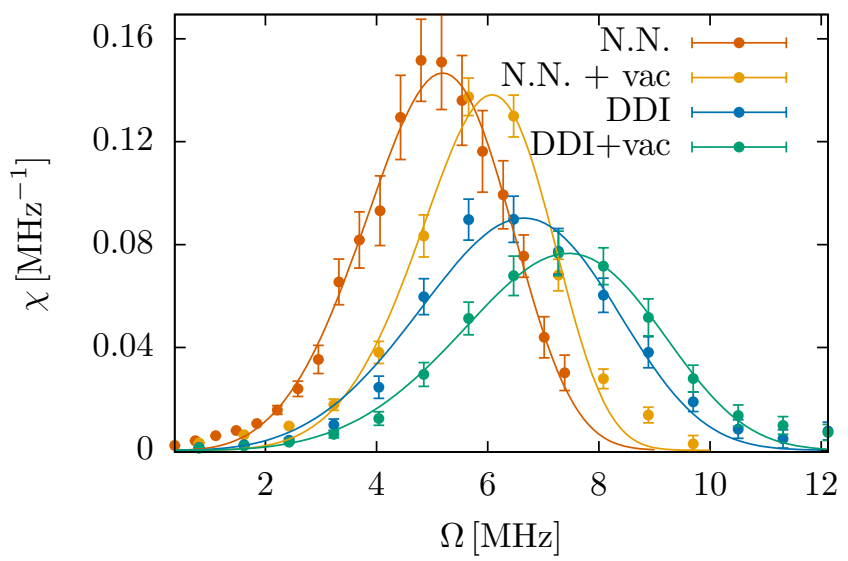

FIG. SM-1. Averaged results from 1000 wave-function Monte-Carlo trajectories showing the steady state susceptibility $\chi$ for different cases of $3 \times 3$ geometry. The orange and yellow lines are for the cases of nearest neighbour interaction (N.N.) and the blue and green lines are for the cases of long-range dipole intearctions (DDI). The yellow and green lines include a vacancy (vac) in the lattice.

In order for the geometries to have comparable interaction energies, we normalize the interaction strength $V_{i j}$ by calculating the total strength for each of the geometries and diving them with that of the nearest-neighbour case. We then run the wave-function Monte-Carlo simulations as discussed earlier and the results are shown in Fig. SM-1. We see that including the long-range interaction and vacancy disorders modify our results only quantitaively and the qualitative findings remain the same.

\section{SENSING IN THE ABSENCE OF AN EXTERNAL BIAS FIELD}

In many applications, it is desirable to perform the sensing without the presence of an external magnetic field $B_{0}$. In this case, the $m_{s}=1$ and $m_{s}=-1$ states are degenerate, and it becomes necessary to include the latter in the analysis. Using the variational principle for open quantum systems [25], we find a very similar behavior of the absolute value of the magnetization of the system, see Fig. SM-2, including again a remarkable robustness to additional $T_{2}$ decoherence processes. Consequently, the dissipative quantum sensor can also be operated with an external bias field. 


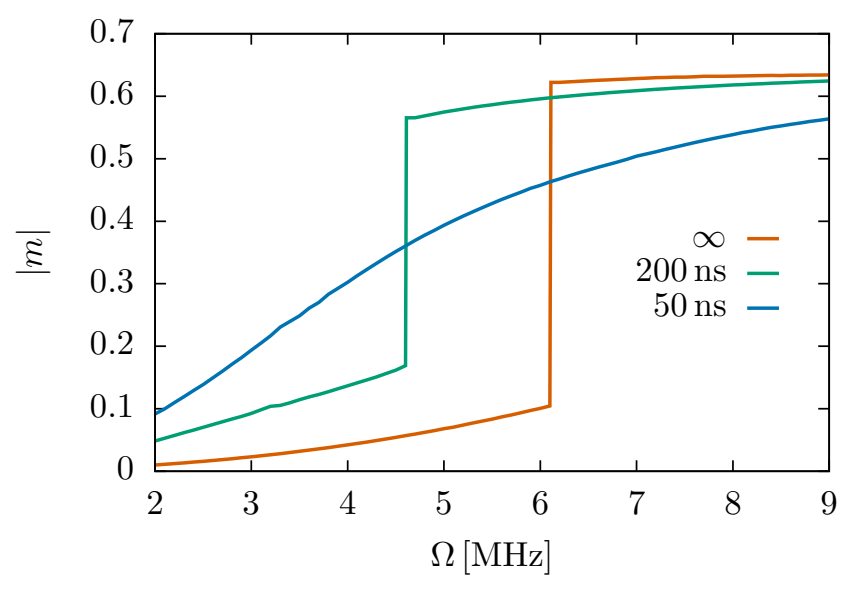

FIG. SM-2. Absolute value of the magnetization of the NV centers in the absence of an external magnetic field. Without additional decohrence processes $(\infty)$ or associated $T_{2}$ times on the order of several hundred nanoseconds, the first order transition required for sensing remains present.

\section{MAGNETIC SUSCEPTIBILITY FOR DC FIELDS}

We calculate the magnetic susceptibility with respect to changes in the magnetic field. As a variation of the magnetic field will shift the resonance condition for the microwave driving of the NV centers, we can capture the effect of a change in magnetic field with respect to the external bias field $B_{0}$ in terms of a finite detuning $\Delta$ in the Hamiltonian (1). Fig. SM-3 shows the magnetic susceptibility $\chi_{D C}=\partial m / \partial \Delta$ as a function of the detuning $\Delta$. As the peak of the susceptibility is only slightly larger than the one for the AC susceptibility, the sensitivities for AC and DC will be comparable.

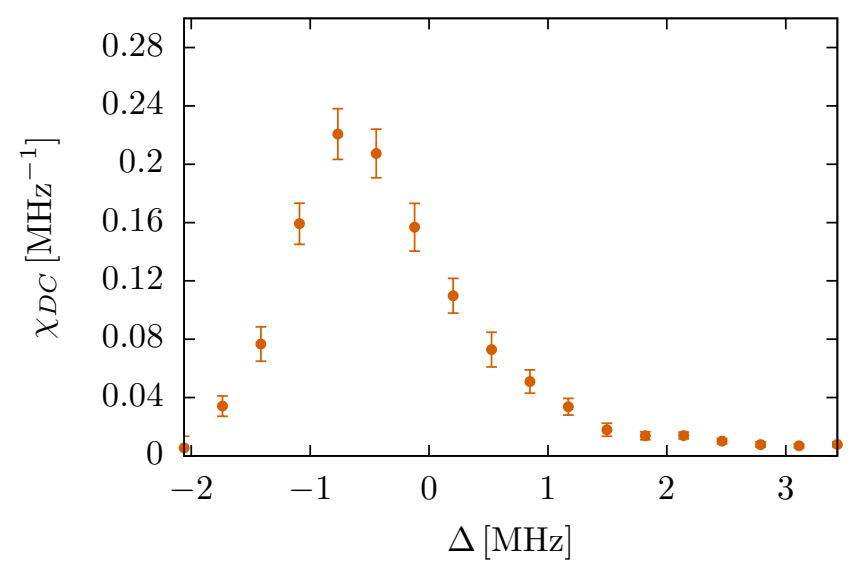

FIG. SM-3. Averaged results from 1000 wave-function Monte-Carlo trajectories showing the steady state susceptibility $\chi_{D C}$ for a $3 \times 3$ geometry.

\section{SENSITIVITY OF THE DISSIPATIVE QUANTUM SENSOR}

In contrast to non-interacting NV sensors, the measurement is not limited by the photon shot noise of the individual NV centers, but rather by the bimodal distribution of the system close to the first order transition. In particular, the signal for the total magnetization of the ensemble behaves as $N\left(m_{0}+\chi_{D C} B\right)$, where $B$ is the external field to be measured. The noise from the bimodal statistics is given in terms of its standard deviation by $N / 2 \delta m$, where $\delta m$ 
is the jump in magnetization at the transition in the thermodynamic limit. From the numerical simulations, we see that the susceptibility behaves essentially like $\sqrt{N}$ close to the transition, leading to an overall $N^{-1 / 2}$ scaling of the sensitivity. The sensitivity is then given by

$$
\eta_{D C}=\frac{\delta m}{2 \chi_{D C} \sqrt{\nu}},
$$

where $\nu$ is the rate of the measurement. For $\delta m=0.3, \nu=1 \mathrm{MHz}$, we obtain $\eta \approx 3 \mathrm{nT} \mathrm{Hz}^{-1 / 2}$ for $N=10^{3}$ spins and $\eta \approx 300 \mathrm{fT} \mathrm{Hz}^{-1 / 2}$ for $N=10^{11}$. 